\title{
MICROFLUIDIC CULTURE MODEL FOR DRUG SCREENING ON SCHISTOSOME PARASITES
}

\author{
Vincent Girod ${ }^{1,2,3}$, Marie-José Ghoris ${ }^{2}$, Jérôme Vicogne ${ }^{2 *}$, Vincent Senez ${ }^{1 *}$ \\ ${ }^{1}$ CNRS, Univ. Tokyo, UMI 2820 - LIMMS, Lille F-59000 \\ ${ }^{2}$ Univ. Lille, CNRS, Inserm, CHU Lille, Institut Pasteur de Lille, U1019 - UMR 9017 - CIIL - \\ Center for Infection and Immunity of Lille, F-59000 Lille, France \\ ${ }^{3}$ Univ. Lille, CNRS, UMR 8520 - IEMN, Villeneuve d'Ascq F-59650 \\ ${ }^{*}$ These authors contributed equally
}

\begin{abstract}
Schistosomiasis is a challenging parasitic model due to the absence of transgenic strains and systems allowing maintenance of heathy worms outside of their host (mammals). Classical in vitro studies (Petri dish) do not allow long-term survival of worm couples with retention of basic biological functions like mobility and pairing. We have designed a microfluidic device that sustainably cultivates adult worm couples. The influences of the composition and physical characteristics of mass flow on the mobility, pairing and long-term survival of worms have been studied. We have also assessed the effect of Praziquantel, the only therapeutic molecule currently in use.
\end{abstract}

\section{KEYWORDS}

Mesenteric vein, Schistosomiasis, flatworms, microfluidic culture of whole animal.

\section{INTRODUCTION}

Schistosomes are helminth parasites that cause schistosomiasis (or Bilharzia), one of the most important parasitic diseases worldwide in terms of mortality and morbidity. According to the World Health Organization, 800 million people are at risk of infection, 230 million are currently infected and schistosomiasis causes about 200 000 deaths per year [1]. The severe pathology of schistosomiasis is due to the remarkable longevity (several decades) of adult worms inside their host associated with their intense and permanent egg production. The reproductive cycle involves two hosts, namely a freshwater snail and a mammal. Asexual reproduction in the snail generates cercariae, the infective stage of the parasite for vertebrate hosts. After being released in water, cercariae penetrate mammalian skin and enter blood and lymphatic capillaries. In this environment, schistosomes continue their transformation into sexually mature male and female worms and finally migrate as paired adult worms to their final habitat: the mesenteric veins. Paired females produce non-embryonated eggs. Embryogenesis process within the host tissues (liver or intestine) and generates a multicellular larva inside the egg, the miracidium. When released in water via faeces, miracidia infect the snail and the reproductive cycle is complete. Eggs that are trapped in host tissues cause the formation of granulomas and elicit inflammatory processes, which affect organ function (i.e.: hepatic fibrosis) and increase the risk of cancer [2]. Praziquantel (PZQ) is the only drug currently used to cure schistosomiasis. It is safe, affordable and effective. However, its widespread use for mass treatment since the early 80's has increased the likelihood of emergence of PZQ-tolerant parasites [3]. In the absence of a vaccine to eradicate schistosomiasis new alternative therapeutics are urgently needed.

Many attempts have been made to improve in vitro culture media to allow the maintenance of a reasonable level of egg production by adult worms but none of them led to hatching of infective miracidia, suggesting that medium optimization is not sufficient [4]. In addition, despite efficient genome editing in eggs, the impossibility of promoting their in vitro embryogenesis impairs the generation of transgenic strains [5]. However, access to transgenic strains would be an unprecedented tool, not only for the identification and validation of therapeutic targets, but also for the spreading of worm strains with compromised phenotypes in endemic areas [6]. Therefore, the development of an instrumented in vitro system that simultaneously promotes adult survival, sufficient egg production and maturation leading to infective miracidia would be a major breakthrough in the fight against schistosomiasis. In addition, such a device would help preclinical screening of new molecules aiming at killing worms but also reducing liver fibrosis.

The study of whole animals in a smart microfluidic system is a very recent research topic in the field of systems biology. Some basic systems have been reported for the study of the free-living $C$. elegans worm, a model that offers convenience, low cost and molecular-genetic tools [7]. Working on whole parasitic species is necessary to identify new therapeutic targets [8] but the scientific literature reports very few studies on trematodes with most focused on the detection of the parasites in human fluid either by microfluidic PCR, antigen identification or egg isolation [9]. The Swiss Tropical and Public Health Institute is developing an 'infected body-on-a-chip' microfluidic platform for antischistosomal drug discovery using electrical impedance spectroscopy for biosensing, while ETH Zurich developed a viability assay designed for the larval schistosomula stage using a microfluidic impedance-based system [10,11]. However, neither of these systems aims at studying adult worm development together with drug effects and preclinical screening.

\section{DESIGN \& FABRICATION}

The geometrical features of the mesenteric vein model for the culture of $S$. mansoni were designed using COMSOL MULTIPHYSICS $\left(\mathrm{Comsol}^{\circledR}\right.$ AB, SE, Stockholm). The device consists of two chambers (Fig. 1AC1) linked by a network of microfluidic channels $200 \mu \mathrm{m}$ in height and various dimensions (between 400 and 100 
$\mu \mathrm{m}$ ) in width, the narrowest reaching the outlet chambers on both sides of the system to prevent worms from escaping the channel network. The worm couples are introduced at the center of the structure and the chambers on each side can collect the eggs. To evaluate the performance of the device, we solved the steady-state Navier-Stokes equation to analyze the flow field and define the right flow rate to ensure that shear stress (between 10 and 20 dynes per $\mathrm{cm}^{2}$ ) is in agreement with the in vivo situation in the mesenteric capillaries [12]. Single chamber chips (200 $\mu \mathrm{m}$ in height, Fig. 1A-C2), based on the diameter of a 6 well Petri dish were developed for fluidic application to compare effects on culture of restrictive or non-restrictive volumes and the presence of medium flow. Simple channel chips (400 $\mu \mathrm{m}$ in height, Fig. 1A-C3) were fabricated to measure adhesion capacity with different surface coatings.

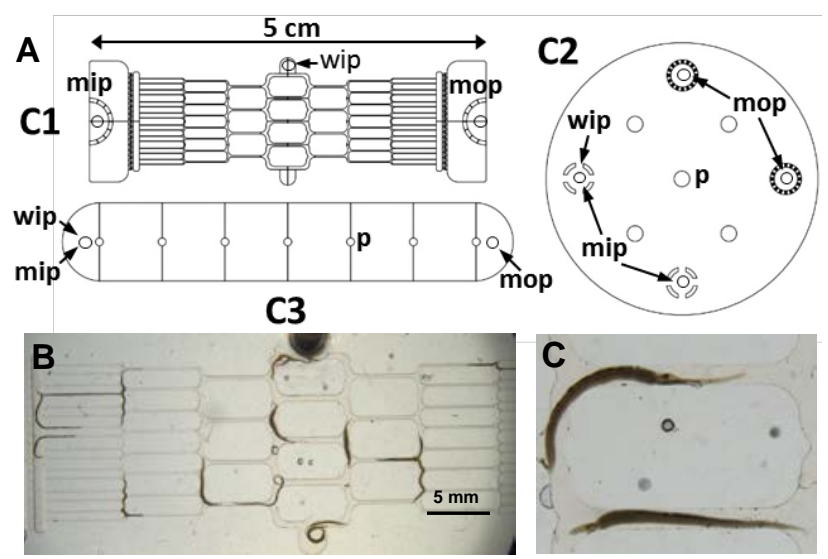

Figure 1: Design of microfluidic chips hosting adult S. mansoni. A: C1 is made of a channel network mimicking the mesenteric veins. C2 has the diameter of a 6 well Petri dish (34.6 mm in diameter (9.6 cm²), $200 \mu \mathrm{m}$ in height) with 5 pillars (p). C3 is a single channel chip (400 $\mu \mathrm{m}$ in height, $5.75 \mathrm{~cm}$ in length, $1 \mathrm{~cm}$ in weight and pillars every $0.7 \mathrm{~cm}$ ). All chips possess media inlet and outlet ports (mip, mop) and a worm inlet port (wip). B: Worm couples circulating in the C1 chip. C: Zoom on paired worms.

The microfluidic devices were fabricated with PDMS $\left(\mathrm{SYLGARD}^{\circledR}, 184\right.$ Silicon Elastomer Kit, Dow Corning, Midland, MI, USA) molding. The master molds were created by SU-8 (KAYAKU ${ }^{\circledR}$, JP) structuration using either standard lithography or back side lithography [13], leading to either square or rounded cross-sections of the mold, the latter having the advantage of being closer to the geometry of blood vessels. The definition of the dimension of the geometry was performed using the layout editor CLEWIN (Phoenix Software ${ }^{\circledR}$, NL, Enschede) and the photomask was homemade. PDMS was prepared with a 10:1 elastomer base-curing agent ratio (w/w), degassed and poured onto the master mold to a thickness of approximately $5 \mathrm{~mm}$. The sample was cured at $95^{\circ} \mathrm{C}$ for one hour. The PDMS channel layer was punched to create inlets and outlets, activated with air plasma and bound to a glass microscope slide to seal the channels. The chips were equipped with appropriate tubing and proper sealing was confirmed after perfusion of water.

\section{EXPERIMENTAL METHOD}

\section{Parasite acquisition and maintenance:}

Golden Syrian hamsters (Mesocricetus auratus) were infested with 2000 cercariae and perfused 40 days post infection to obtain adult worm pairs [15]. Worms were washed in RPMI (RPMI 1640 GIBCO $^{\circledR}$, UK, 61870-010) and maintained in an incubator $\left(37^{\circ} \mathrm{C}\right.$, humid atmosphere, $5 \% \mathrm{CO}_{2}$ ) in standard RPMI supplemented with heatinactivated Horse serum (10\%) $\left(\mathrm{GIBCO}^{\circledR}\right.$, UK, 16050 122), rifampicin (60 $\mu \mathrm{g} \cdot \mathrm{mL}^{-1}$ in $\left.\mathrm{DMSO}\right)$ (EUROMEDEX $\left.{ }^{\mathrm{TM}}, \mathrm{FR}, 1059\right)$, penicillin (50 units.mL $\mathrm{m}^{-1}$ )

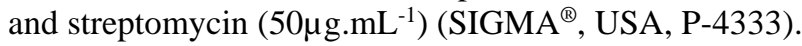

\section{Culture in different media:}

Duplicate groups of 10 couples $(n=4)$ were incubated in 6 well plates $\left(9.6 \mathrm{~cm}^{2}\right)$ with $3 \mathrm{~mL}$ of different combinations of media M199 (GIBCO ${ }^{\circledR}$, UK, 41150-020), DMEM (GIBCO ${ }^{\circledR}$, UK, 61965-026) and RPMI 1640 (GIBCO $\left.^{\circledR}, \mathrm{UK}, 61870-036\right)$ supplemented or not with fetal calf serum (PAA ${ }^{\circledR}$, AT, A15-101), horse serum or human serum (Institute Pasteur of Lille, FR). In all combinations, media were supplemented with antibiotics, as described above. Medium combination effects were analyzed by evaluating worm heath status (S2 = normal movement and normal tissue aspect; $\mathrm{S} 1$ = reduced movement or degraded tissue; S0 =dead, no movement) every others day before media renewal $(2 \mathrm{~mL})$. RStudio (RStudio ${ }^{\circledR}$, AT, Vienna) was used to correct observational error between S1 and S0 status, normalize the data and calculate standard deviations for each combination.

\section{Microfluidic and Petri culture:}

Tubing length was calculated to generate a dead volume of $1 \mathrm{~mL}$. About 2 days before worm introduction, microfluidic devices (C1 and C2) were connected to a peristaltic pump (ISMATEC ${ }^{\circledR}, \mathrm{CH}$, IPS-N4 ISM935) and perfused with $5 \mathrm{~mL}$ of ethanol $70 \%$, rinsed twice with $5 \mathrm{~mL}$ of $\mathrm{H}_{2} \mathrm{O}$ and initiated with $5 \mathrm{~mL}$ of RPMI supplement with horse serum (RPMI/Horse) at $0.1 \mathrm{~mL} / \mathrm{min}$ flow rate. Then, input and output were connected to the same tank and all media were immediately changed with $4 \mathrm{~mL}$ of RPMI/Horse. Devices were immersed in $\mathrm{H}_{2} \mathrm{O}$ inside the incubator. Quadruplicates of 10 couples were incubated twice in C1, C2 and Petri dish. Culture effect measurements and data treatments were executed as described above before replacement with $4 \mathrm{~mL}$ of media.

\section{Worm adhesion assay on coated surfaces:}

Madin-Darby Canine Kidney cells (MDCK) were cultured in DMEM media supplemented with heatinactivated fetal bovine serum (10\%) $\left(\mathrm{GIBCO}^{\circledR}\right.$, USA, 10270-106), non-essential amino acids $\left(\right.$ GIBCO $^{\circledR}$, USA, 11140-050), biotin (SIGMA ${ }^{\circledR}$, USA, B4639) and $5 \mathrm{~mL}$ of ZellScheild $^{\circledR}$ (DE, 13-0150). C3 coated chips were prepared with $50 \mu \mathrm{g} / \mathrm{mL}$ of collagen (Cellmatrix Type I-A, FUJIFILM $^{\circledR}$, DE, 631-00651) in acetic acid 0.02M for $2 \mathrm{~h}$, rinsed twice with $3 \mathrm{~mL}$ of Phosphate Saline Buffer $1 \mathrm{X}$ (PBS) and dried at RT. C3 chips were initiated with $10 \mathrm{~mL}$ of MDCK cell culture media. MDCK cells (10.000 cells/chip) were introduced into the chip chamber and placed inside the incubator without flow rate for $3 \mathrm{~h}$. Unattached cells were flushed at a $35 \mu \mathrm{L} / \mathrm{min}$ flowrate for 
48 h. Next, 30 couples were introduced for each type of C3 and recorded $\left(\mathrm{NIKON}^{\circledR}\right.$, JP, SMZ18, Camera DS-FI3, Objectif P2-SHR plan APO 0.5X, Zoom 0.75X). Flow rate was increased $(50 \mu \mathrm{L} / \mathrm{min}$ to $300 \mu \mathrm{L} / \mathrm{min}$ by $50 \mu \mathrm{L} / \mathrm{min}$ steps each minute, $300 \mu \mathrm{L} / \mathrm{min}$ to $1 \mathrm{~mL} / \mathrm{min}$ by $100 \mu \mathrm{L} / \mathrm{min}$ steps every 2 minutes and $1 \mathrm{~mL} / \mathrm{min}$ to $3.4 \mathrm{~mL} / \mathrm{min}$ by 200 $\mu \mathrm{L} / \mathrm{min}$ steps every 3 minutes). Worm exits were optically counted on recorded videos. Finally, a normalization of the data was applied using RStudio.

\section{Microfluidic evaluation of praziquantel (PZQ):}

PZQ (SIGMA ${ }^{\circledR}$, USA, P-4668) was prepared as a $0.5 \mathrm{M}$ stock solution in DMSO and then diluted from 400 to $30 \mathrm{nM}$ in RPMI Horse. C3 chips were coated with collagen and initiated with RPMI/Horse media as described above. Quadruplicates of 30 couples were introduced at 50 $\mu \mathrm{L} / \mathrm{min}$ flowrate and record by SMZ. Flow rate was increased to $1 \mathrm{~mL} / \mathrm{min}$ during $1 \mathrm{~min}$ and PZQ was added after $5 \mathrm{~min}$. Worms exiting the chips were counted and data was normalized every 5 minutes using RStudio.

\section{RESULTS \& DISCUSSION}

The modification of cell culture media to maintain paired and healthy adult worms has been a long-lasting quest in the field. Composition of the basal medium and the source of serum seem to be the main parameters conditioning worm survival $[4,14]$. However, due to many discrepancies in the results obtained by deferent labs, we first determined the best combinations in our hands.

After one month of culture, we showed that serum origin has a major impact on worm survival and, as expected, the absence of serum is quickly deleterious (Fig. 2 ). Although calf serum is the most commonly used serum for parasite culture, human and horse serum gave a net advantage. Medium composition is less critical but RPMI gives a slightly better survival rate whatever the serum used. Therefore, we selected the combination of RPMI medium and horse serum, which has an easier and safer access compared to human serum, for subsequent experiments.

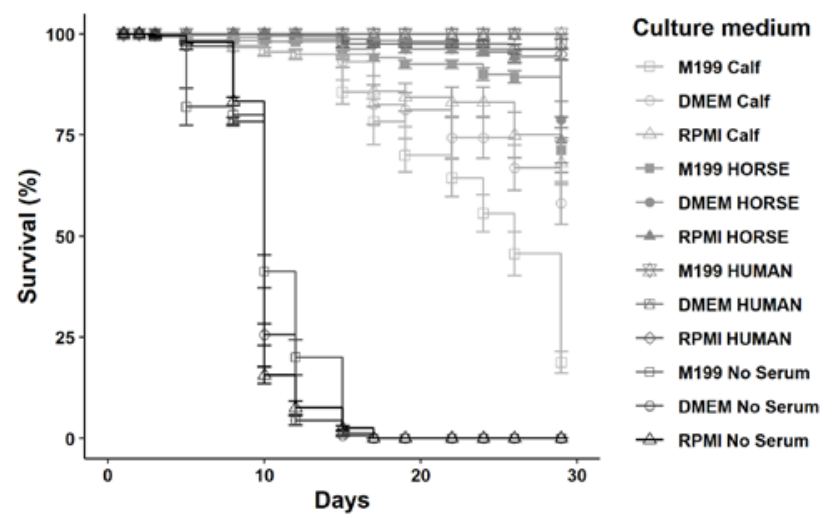

Figure 2: Evolution of the survival rate of Schistosome pairs as a function of time for different culture media in static Petri dish culture conditions.

Next, we evaluated the effect of the microfluidic environment on worm survival. Indeed, generating a continuous medium flow could optimize media renewal around worms but could also require additional efforts from the worms leading to their exhaustion. Therefore, we compared worm survival in a regular 6 well Petri dish, in a vascular mimetic chip (C1) and in large chip with the size of a Petri dish (C2) for 25 days (Fig. 3). Our results demonstrate that culture in a microfluidic environment has no detrimental effect on worm survival, but surprisingly, worm pairing is well maintained in both systems (Fig. 3).

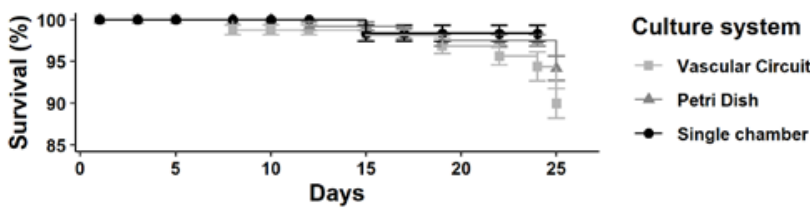

Figure 3: Survival rate of schistosomes as a function of time for different culture models: vascular chip (C1), regular Petri dish and single chamber chip (C2).

Having demonstrated that microfluidic culture allows long lasting culture and has a beneficial effect on worm pairing, we evaluated the effect of surface coating on worm attachment. Worms use oral and ventral suckers to attach to surfaces. Males are also covered by spiky tubercles that could favor attachment or crawling on surfaces. Thus, we coated a long single channel chip (C3) with collagen, MDCK cells or both and determined worm attachment ability depending on media flowrates (Fig. 4).

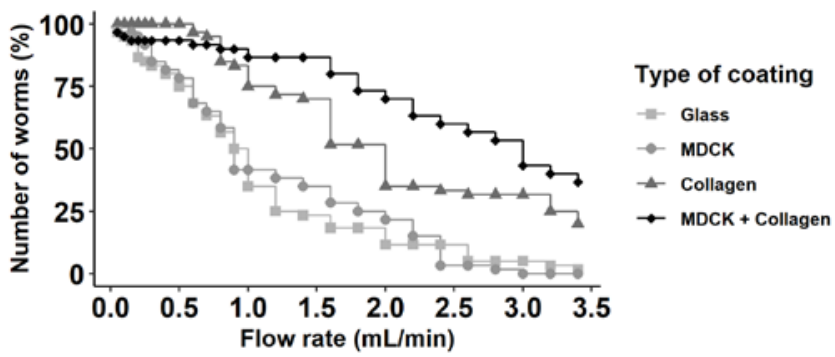

Figure 4: Percentage of Schistosomes attached to chip surface as a function of flow rate and surface coatings: glass, MDCK cells, collagen and collagen with MDCK cells.

This experiment demonstrates that surface treatments have major impacts on worm attachment. A glass surface does not allow a good attachment of the worms as soon as the flow rate exceeds $50 \mu \mathrm{L} / \mathrm{min}$. MDCK cells alone do not improve attachment since it seems that worms peel them off the surface in the absence of collagen treatment. In contrast, collagen and a top layer of MDCK cells strongly promote worm attachment. This coating probably favors the grip of worm suckers on a softer and flexible surface compared to glass.

Finally, we analyzed the ability of worms to stay attached in a collagen coated C3 chip when exposed to PZQ (Fig. 5). When untreated, a $1 \mathrm{~mL} / \mathrm{min}$ flow rate allowed a $>75 \%$ worm retention in the chips for at least one hour. The average IC 50 value of praziquantel is $750 \mathrm{nM}$ and a dose $>200 \mathrm{nM}$ is necessary to observe a measurable lethal effect after $120 \mathrm{~h}$ in a standardized and optimized in vitro assay [15]. In our device, we observed a direct effect on worm attachment at concentrations as low as $50 \mathrm{nM}$ with no noticeable morphological changes. A 400 nM concentration induces a rapid detachment of worms. 


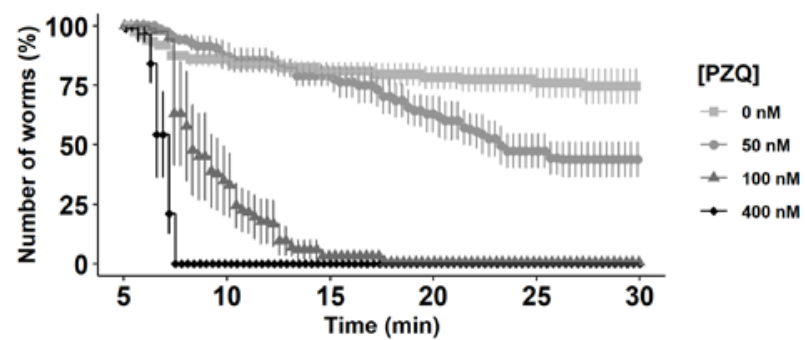

Figure 5: Percentage of worms attached in the chip in presence of PZQ as function of time at a $1 \mathrm{~mL} / \mathrm{min}$ flowrate.

\section{CONCLUSION}

In this report, we demonstrate for the first time that long-lasting maintenance of adult schistosome couples in a microfluidic device is feasible, giving access to classical parameters like mobility and pairing but also to additional ones like motility and attachment capabilities, compared to classical in vitro studies. We show that worms can survive in a media flow in a single channel but also in a branched channel system, mimicking the mesenteric vein architecture. We also demonstrated that, in a fluidic environment, surface coating has a critical effect on worm adhesion and motility. A simple collagen coating is sufficient to improve gripping by oral and ventral suckers of males and females but a cell coating gives a stronger support even with very high flow rates.

Interestingly, a major effect of PZQ on worm motility and surface adhesion is observed at doses as low as $50 \mathrm{nM}$, which are not considered significant or lethal in regular in vitro assays. Therefore, the fluidic system could represent a new tool to test drugs devoid of an immediate lethal effect noticeable in vitro but that could induce "worm flushing out" in vivo.

Our next goal will be to study worm longevity and fertility parameters in the different fluidic systems but also to evaluate the effect and measure the IC50 of current (PZQ, Artemisinin, Oxamniquine) or new drugs [16] in comparison with traditional in vitro assays.

\section{ACKNOWLEDGEMENTS}

We would like to thank I-Site Lille, CPER Haut de France and Institut Pasteur de Lille for financial supports. We thank SMMILE for providing microfabrication and imaging equipment and Raymond Pierce for proofreading.

\section{REFERENCES}

[1] D. G. Colley, A. L. Bustinduy, W. E. Secor, et C. H. King, « Human schistosomiasis ", The Lancet, vol. 383, nº 9936, p. 2253-2264, juin 2014.

[2] M. H. Mostafa, S. A. Sheweita, et P. J. O’Connor, "Relationship between Schistosomiasis and Bladder Cancer », Clin. Microbiol. Rev., vol. 12, n 1, p. 97-111, janv. 1999, doi: 10.1128/CMR.12.1.97.

[3] M. J. Doenhoff, D. Cioli, et J. Utzinger, « Praziquantel: mechanisms of action, resistance and new derivatives for schistosomiasis ", Curr. Opin. Infect. Dis., vol. 21, n ${ }^{0}$ 6, p. 659-667, déc. 2008, doi: 10.1097/QCO.0b013e328318978f.

[4] J. Wang, R. Chen, et J. J. Collins, « Systematically improved in vitro culture conditions reveal new insights into the reproductive biology of the human parasite Schistosoma mansoni », PLOS Biol., vol. 17, $\mathrm{n}^{0}$ 5, p. e3000254, mai 2019, doi:

10.1371/journal.pbio.3000254.

[5] W. Ittiprasert et al., « Programmed genome editing of the omega-1 ribonuclease of the blood fluke, Schistosoma mansoni », eLife, vol. 8, 15 2019, doi: 10.7554/eLife.41337.

[6] P. Cai, G. N. Gobert, H. You, et D. P. McManus, « The Tao survivorship of schistosomes: implications for schistosomiasis control », Int. J. Parasitol., vol. 46, $\mathrm{n}^{0}$ 7, p. 453-463, 2016, doi: 10.1016/j.ijpara.2016.01.002.

[7] S. K. Gokce et al., « A multi-trap microfluidic chip enabling longitudinal studies of nerve regeneration in Caenorhabditis elegans », Sci. Rep., vol. 7, n 1, Art. $\mathrm{n}^{\mathrm{0}}$ 1, août 2017.

[8] J. C. Weeks et al., « Microfluidic platform for electrophysiological recordings from host-stage hookworm and Ascaris suum larvae: A new tool for anthelmintic research », Int. J. Parasitol. Drugs Drug Resist., vol. 6, nº 3, p. 314-328, déc. 2016.

[9] M. M. Nigo, G. Salieb-Beugelaar, M. Battegay, P. Odermatt, et P. Hunziker, « Schistosomiasis: from established diagnostic assays to emerging micro/nanotechnology-based rapid field testing for clinical management and epidemiology », Precis. Nanomedicine, vol. 3, $\mathrm{n}^{\circ}$ 1, p. 439-458, déc. 2019, doi: 10.33218/prnano3(1).191205.1.

[10] M. M. Modena et al., « Impedance-based detection of Schistosoma mansoni larvae viability for drug screening ", in 2017 IEEE Biomedical Circuits and Systems Conference (BioCAS), oct. 2017, p. 1-4, doi: 10.1109/BIOCAS.2017.8325227.

[11] K. Chawla et al., « Impedance-based microfluidic assay for automated antischistosomal drug screening », ACS Sens., vol. 3, n 12, p. 2613-2620, déc. 2018, doi: 10.1021/acssensors.8b01027.

[12] T. Papaioannou et C. Stefanadis, « Vascular Wall Shear Stress: Basic Principles and Methods », Hell. J. Cardiol. HJC Hellēnikē Kardiologikē Epitheōrēsē, vol. 46, p. 9-15, janv. 2005.

[13] M. Fenech, V. Girod, V. Claveria, S. Meance, M. Abkarian, et B. Charlot, « Microfluidic blood vasculature replicas using backside lithography », Lab. Chip, vol. 19, n ${ }^{\circ} 12$, p. 2096-2106, 2019, doi: 10.1039/C9LC00254E.

[14] P. F. Basch, « Cultivation of Schistosoma mansoni in vitro. II. production of infertile eggs by worm pairs cultured from cercariae », J. Parasitol., vol. 67, n 2, p. 186-190, avr. 1981.

[15] B. Ramirez, Q. Bickle, F. Yousif, F. Fakorede, M.-A. Mouries, et S. Nwaka, « Schistosomes: challenges in compound screening », Expert Opin. Drug Discov., vol. 2, nº sup1, p. S53-S61, oct. 2007, doi: 10.1517/17460441.2.S1.S53.

[16] M. Vanderstraete et al., « Dual Targeting of Insulin and Venus Kinase Receptors of Schistosoma mansoni for Novel Anti-schistosome Therapy ", PLoS Negl. Trop. Dis., vol. 7, $\mathrm{n}^{0}$ 5, p. e2226, mai 2013. 